\title{
Perioperative Complications in Obstructive Sleep Apnea Patients
}

\author{
Dennis Auckley ${ }^{1}$
}

Published online: 6 April 2016

(C) Springer International Publishing AG 2016

\begin{abstract}
Obstructive sleep apnea (OSA) is growing in prevalence though it still remains significantly underdiagnosed. Preoperative screening for OSA is taking place in many institutions, with a substantial number of patients being identified as having a high probability for OSA just prior to undergoing surgery. Emerging data increasingly demonstrates that patients with a high probability of having OSA as well as those with a known diagnosis of OSA may be at increased risk for developing complications in the perioperative setting. Postoperative cardiac and pulmonary adverse events appear to occur more frequently in these patients though the impact OSA has on postoperative mortality is controversial. In addition, determining which patients are at greatest risk remains an area of uncertainty. This chapter will review the current state of the knowledge regarding OSA as a risk factor for perioperative complications.
\end{abstract}

Keywords Sleep apnea $\cdot$ Obstructive sleep apnea $\cdot$ OSA . Perioperative complications · Postoperative complications

\section{Introduction}

Obstructive sleep apnea (OSA) is increasingly common with the most recent prevalence estimates for moderate to severe

This article is part of the Topical Collection on Sleep Related Breathing Disorders

Dennis Auckley

dauckley@metrohealth.org

1 The Division of Pulmonary, Critical Care, and Sleep Medicine, MetroHealth Medical Center, Case Western Reserve University, 2500 MetroHealth Drive, Cleveland, OH 44109, USA
OSA in individuals 30-70 years old at $6 \%$ in women and $13 \%$ in men [ $[\bullet]$. Despite increasing awareness of this disorder, the majority of individuals with OSA remain undiagnosed $[2,3]$. OSA has been associated with numerous long-term health consequences, including cardiovascular, metabolic, and cognitive impairments, as well as increased accident rates and lower quality of life [4]. Recently, more acute clinical consequences related to OSA have garnered interest, particularly regarding the impact of OSA on outcomes in individuals subjected to the surgical environment, where a multitude of perioperative factors, including anesthetics and opioids, can affect breathing. Initially, case series in the 1990s [5, 6] described postoperative respiratory complications in patients with OSA. Subsequently, a number of controlled studies have found a range of perioperative complications to be associated with a diagnosis of OSA, though the strength of the associations and the quality of data vary considerably.

This review will discuss the diagnosis of OSA in the preoperative setting, the prevalence of OSA in surgical populations, mechanisms by which OSA might increase the risk for perioperative complications, and specific perioperative complications that have been associated with known or suspected OSA. Complications associated with surgery performed for the management of OSA, as well as pediatric surgery in children with known or suspected OSA, will not be reviewed as these are beyond the scope of this article.

\section{Defining OSA in the Perioperative Setting}

In perioperative studies, OSA has been defined by sleep studies, questionnaires, chart diagnoses, and International Classification of Diseases (ICD) codes. Understanding the advantages and disadvantages of these different methodologies is helpful when assessing the quality of the studies and their conclusions. 
In addition to having symptoms typical of OSA, the diagnosis of OSA requires objective testing. An attended polysomnogram (PSG) performed in a sleep laboratory has been considered the gold standard to determine the apneahypopnea index (number of apneas and hypopneas per hour of sleep $=\mathrm{AHI}$ ), the index used to define the presence of OSA and determine its severity. An AHI $\geq 5$ events per hour of sleep, in the setting of the OSA symptoms, is the minimal criterion used for diagnosing OSA [7]. Consensus severity grading of OSA suggests mild OSA $=$ AHI $5<15$ events per hour, moderate OSA $=$ AHI $15<30$ events per hour, and severe $\mathrm{OSA}=\mathrm{AHI} \geq 30$ events per hour [8]. While PSG is the gold standard for diagnosing OSA, limitations to access and costs associated with the procedure have limited PSG-defined perioperative OSA studies to being primarily retrospective.

Portable limited-channel sleep studies, or home sleep apnea testing (HSAT) devices, are an acceptable alternative to PSG for the diagnosis of OSA [9]. HSAT devices measure a limited number of parameters related to breathing but usually do not have sensors to accurately measure sleep. As a result, the number of respiratory events is divided by hours of monitoring time [10], not hours of sleep, and thus, the AHI (called the respiratory event index, or REI, for HSAT studies) may underestimate the true AHI, resulting in a nontrivial falsenegative rate (as high as $19 \%$ ) [11]. These devices have advantages of ease of use, enhanced access, and less expense as compared to a PSG. However, many devices either have not been validated against PSG or have only been validated in patients without significant medical co-morbidities. In addition, HSAT devices also carry a small but significant technical failure rate. HSAT devices have been used to evaluate for OSA in the preoperative setting [12-14], and unattended full PSG done in the home has been successfully used in a couple of studies $[14,15]$ though this approach requires significant resources to successfully implement.

Overnight oximetry is another tool that has been used to screen for or to risk stratify OSA. Overnight oximetry provides data on oxygenation and heart rate and can generate an oxygen desaturation index (ODI) as well as assess for the presence of hypoxemia. It has the advantages of simplicity, widespread availability, and low cost. When compared to PSG where desaturations are required to score respiratory events, oximetry has an accuracy of 86-94\% for detecting moderate to severe OSA [16, 17]. However, respiratory events are not always associated with desaturations [10], and thus, clinically significant OSA may be missed by oximetry. Thus, the ODI may also underestimate the AHI. In addition, interpretations of oximetry are not standardized and often highly variable [18]. Overnight oximetry cannot be used to formally establish a diagnosis of OSA for the purposes of prescribing CPAP therapy. Despite these limitations, oximetry may have some utility for risk stratifying patients preoperatively $[19,20]$.
A variety of screening questionnaires have been used to identify patients at high risk for OSA and used as surrogates for diagnosing OSA in some perioperative studies [21-29]. The accuracy of screening questionnaires has been assessed in recent reviews $[30,31]$ and is highly variable, reflecting different testing populations (i.e., high versus low prevalence of disease) and different diagnostic criteria used for defining OSA. In general, most of the screening tools (STOP questionnaire, STOP-Bang questionnaire, Berlin questionnaire, Sleep Apnea Clinical Screen (SACS) questionnaire, American Society of Anesthesiology (ASA) checklist) have been found to have reasonable sensitivities (70-85\%) and positive predictive values (72-90\%) but poor specificities (40-55\%) and negative predictive values (40-60\%). These findings indicate that screen-positive patients are likely to have OSA but that screen-negative patients may still have OSA and thus confound controlled studies. Of all the screening tools available, the STOP-Bang questionnaire is most widely used, likely due to its relative ease of use and additional potential discriminating value for classifying severity of OSA [32, 33]. A recent meta-analysis of the STOP-Bang questionnaire has confirmed its accuracy in surgical patients [34].

Chart diagnosis and ICD coding of OSA have been used in large database studies to associate OSA with perioperative outcomes. While this technique enables investigators to evaluate sizable populations, the accuracy of these methods has recently been called into question [35]. Examining a database from an academic health sciences network of nearly 5000 preoperative patients, researchers found the ICD-9 diagnosis of OSA to be $58 \%$ sensitive and $38 \%$ specific, suggesting caution be applied when interpreting studies utilizing administrative codes.

\section{OSA Severity Assessment Preoperatively}

Severity of OSA as defined by AHI or REI has not been shown to correlate with risk for postoperative complications. However, a higher ODI (ODI $4 \%>28.5$ events per hour) predicted patients at highest risk for postoperative complications in one study [36]. Questionnaires have generally labeled patients as high or low risk for OSA, though the STOP-Bang questionnaire may be useful for differentiating mild from moderate to severe OSA $[32,33]$. How this correlates with risk for postoperative adverse events is uncertain.

\section{Role of Screening for or Identifying OSA Preoperatively}

The goal of preoperative screening for and/or diagnosis of OSA should be to identify patients most likely to develop postoperative complications in hopes of reducing their risk via care pathways. Aside from overnight oximetry parameters [20], another approach has examined utilizing a combination of screening questionnaire (SACS) and recurrent 
postanesthesia care unit (PACU) events (bradypnea, apneas, desaturations, and/or pain-sedations mismatch) to identify patients most likely to experience respiratory problems following surgery [21]. In a prospective study, the rate of postoperative oxygen desaturations and respiratory complications were considerably higher (33\%) in those screening high risk for OSA preoperatively who also had recurrent PACU events as compared to those screening low risk for OSA with no recurrent PACU events (1\%) [21]. Additional data of this nature is needed.

Identifying patients at risk for OSA in the surgical population may also offer long-term health benefits. Screening for OSA in presurgery clinics can detect patients at high risk for OSA who might otherwise go undiagnosed. Data suggests that diagnosing and treating OSA improves a variety of outcomes, including quality of life and cardiovascular morbidity and mortality [36, 37]. In support of this, a survey study of patients screening high risk for OSA preoperatively, who subsequently underwent confirmatory PSG and treatment, found that at 2 years the patients had improved quality of life and reduced use of medications for co-morbidities [38].

\section{Prevalence of OSA in Surgical Populations}

The prevalence of OSA in the adult population is estimated at $6-13 \%[1 \bullet]$, though significantly higher rates can be found in patients with type II diabetes, polycystic ovarian disease, and cardiovascular diseases [39]. Patients presenting for elective surgery appear to have higher prevalence rates of OSA than the general population, as supported by limited data. Screening questionnaires have found between 24 and $48 \%$ of patients presenting for elective surgeries are at risk for OSA $[12,27,40]$. Acknowledging potential bias due to pretest screening and small numbers, studies utilizing HSAT or PSG preoperatively show that between 20 and $73 \%$ of preoperative patients meet objective criteria for OSA [12, 41, 42]. Patients undergoing evaluation for bariatric surgery, often morbidly obese, represent a specific population of interest. Indeed, studies where PSG or HSAT were systematically completed in all patients have found prevalence rates of OSA to be 70-90\% [43-46], suggesting objective sleep testing should be part of the routine assessment prior to bariatric surgery.

The vast majority (70-87\%) of elective surgery patients who have OSA have not been diagnosed with OSA at the time of their presentation $[12,16]$. These patients may be particularly vulnerable to postoperative complications as strategies for risk reduction would not be implemented due to the unrecognized nature of their OSA. Relying on clinicians to routinely detect patients at risk for OSA in the preoperative setting has been shown to be unreliable [13]. In a study of 267 patients presenting for elective surgery who had a preoperative PSG diagnosing moderate to severe OSA, $92 \%$ of surgeons and $60 \%$ of anesthesiologists failed to consider OSA as a diagnosis in these patients. Many institutions are now using preoperative screening protocols to detect patients at high risk for OSA.

\section{How OSA Contributes to Perioperative Complications}

\section{Mechanisms for Intraoperative Complications}

In OSA patients, the upper airway is often crowded due to excessive adipose tissue or narrowed as the result of craniofacial abnormalities, leading to poor visualization. Add in the use of sedatives and induction anesthetics, which can exacerbate collapsibility of the upper airway [47], and airway management can become difficult. The effects of sedatives and anesthetics on the upper airway of OSA patients were systematically reviewed, and it was found that, while they can lead to adverse events during intubations, the frequency is low [48•]. However, the overall quality of the literature was felt to be poor and better data is needed. The use of topical anesthetics during intubation may also impair protective upper airway reflexes and attenuate genioglossus phasic activity, both of which can precipitate apnea during induction [49, 50]. Patients with OSA frequently have co-morbid gastroesophageal reflux disease which may increase risk of aspiration during induction of general anesthesia, and thus, aspiration prophylaxis has been recommended [51].

\section{Mechanisms for Postoperative Pulmonary Complications}

Numerous factors may contribute to apnea and worsening pulmonary function following surgery. Supine positioning, often required following surgery, may worsen OSA in half of OSA patients [52]. In addition, the combination of obesity and supine positioning may lead to decrements in lung volume, increasing the propensity for upper airway collapse [53] as well as worsening gas exchange [54]. Residual effects of anesthetics can further aggravate decrements in functional residual capacity and oxygenation, potentially leading to more apneic events and hypoxemia [55]. In addition, anesthetic agents decrease upper airway muscle activity and depress the arousal response, further aggravating OSA [47].

Opioids, used both intraoperatively and postoperatively, have a myriad of effects on ventilation, sleep apnea, and sleep [56••]. Opioid-induced ventilatory impairment (OIVI) is an uncommon but potentially catastrophic complication of opioid therapy that can be fatal. Populations considered to be at higher risk for OIVI include the morbidly obese and those with OSA [56・*]. Two recent studies highlight the association between OIVI and OSA, finding in a database analysis that $30 \%$ of patients with postoperative opioid-related life- 
threatening respiratory events had OSA [57] and that $40 \%$ of postoperative opioid-related deaths in an ASA closed claims registry analysis had known or suspected OSA [58]. Opioids also decrease genioglossus muscle activity and suppress the arousal response to apneas and hypopneas [59], resulting in more frequent and prolonged obstructive events during sleep. These effects are variable, however, and the underlying OSA phenotype may determine susceptibility. Opioids can also increase central apneic events in some individuals [60] though this has not been consistently documented in the postoperative setting [61].

Opioids also alter sleep architecture, suppressing REM and slow-wave sleep while increasing sleep fragmentation [56••, $60]$. There is a growing line of evidence suggesting that sleep fragmentation and/or sleep deprivation in and of themselves can lead to hyperalgesia [56••]. In addition, recent work has indicated that intermittent nocturnal hypoxemia may also increase pain reporting [62] and that treating OSA with CPAP may reduce pain sensitivity [63]. These latter findings may have direct implications regarding the use of opiates in OSA patients following surgery.

Anywhere from 10 to $36 \%$ of OSA patients experience REM-predominant sleep apnea [64]. REM sleep may be significantly reduced or absent on the first postoperative night due to the effects of medications, pain, and/or frequent patient care interventions [61]. As a result, REM-rebound sleep may occur on the second to third nights following surgery, resulting in an increase in the number and severity of respiratory events $[65,66]$. However, this is not a uniform finding in all OSA patients [61].

OSA, independent of obesity, has been associated with a hypercoagulable state, and this may be one of the mechanisms by which OSA affects long-term cardiovascular health [67], and could potentially increase thromboembolic disease risk in OSA patients in the postoperative setting.

\section{Mechanisms for Postoperative Cardiac Complications}

The sleep disruption, hypoxemia, and hypercapnia that accompany breathing disturbances during sleep can lead to repeated increases in both sympathetic and parasympathetic tone, adversely impacting cardiopulmonary function [68]. Aside from elevations in nocturnal blood pressure, a variety of arrhythmias including bradycardia, prolonged sinus pauses, second-degree heart block, and atrial and ventricular tachyarrhythmias have been shown to occur at a higher frequency in OSA patients $[68,69]$. The pattern of nocturnal sudden cardiac death described in OSA patients may in part be related to these mechanisms [70]. In the postoperative setting, worsening of the underlying OSA may enhance these cardiovascular changes and potentially predispose patients to higher rates of adverse cardiac events.

\section{Mechanisms for Neurocognitive Complications}

OSA has been associated with neurocognitive dysfunction. Sleep fragmentation is thought to lead to attention/vigilance dysfunction while global cognitive dysfunction may result from intermittent nocturnal hypoxemia [71]. OSA has been suggested to be a possible predisposing factor for delirium [72]. More specifically, chronic microvascular ischemia and chronic inflammation induced by OSA-related sleep disruption and intermittent hypoxemia, coupled with the acute stress of the perioperative environment, including the use of central nervous system-altering medications, have been proposed as possible mechanisms contributing to postoperative delirium $[73,74]$.

\section{OSA Phenotypes}

The majority of patients with OSA will not experience postoperative complications suggesting that there may be interindividual differences affecting susceptibility. Recent interest has focused on variability in arousal thresholds and how this may affect a patient's clinical response to different situations [75]. For example, in patients with low arousal thresholds where repeated respiratory-related arousals tend to fragment sleep, which may lead to subsequent respiratory events, mild sedation could potentially attenuate this response [75]. Conversely, in patients with a high respiratory-related arousal threshold, the addition of sedatives and/or opiates may further increase the arousal threshold leading to prolonged respiratory events with worsening hypoxemia and hypercapnia, theoretically placing the patient at risk for adverse events $[56 \bullet \bullet, 75]$. It is the latter group who might be most susceptible to complications in the postoperative setting, though arousal thresholds have not been studied in postoperative patients yet.

Interindividual variability may also exist in propensity for airway collapse (as measured by critical closing pressure and genioglossus muscle activity) and loop gain of the ventilatory system [76]. It may be that a certain OSA phenotype (i.e., more collapsible airway, higher arousal threshold) is prone to run into problems when subjected to the many factors affecting sleep and breathing in the postoperative environment. Further study is needed to determine if this is relevant.

\section{OSA and Perioperative Complications}

A sufficient number of controlled trials have now been performed such that two meta-analyses and two systematic reviews have examined this topic in detail [77-79, 80••]. The most comprehensive, and most recent, of these reviews analyzed 61 studies and included over 400,000 OSA patients (due to the inclusion of large database studies) [80••]. The risk for complications varies depending on the intensity of anesthesia 
and location of surgery (outpatient versus inpatient) as well as by the specific complication studied. It should be noted that research has only included elective procedures and nonemergent surgery.

\section{Procedures with Conscious Sedation}

Initial concern was raised about increased intraprocedural complications (hypoxemia and airway maneuvers) in patients at high risk for OSA undergoing conscious sedation for endoscopic procedures [81]. However, subsequent studies have not born this out. In a prospective study that screened patients $(n=904)$ for OSA risk prior to endoscopic procedures requiring conscious sedation, no difference was found in complication rates by OSA risk stratification [82]. Additional retrospective studies totaling over 2100 endoscopic procedures under conscious sedation reported no increase in intra- or postprocedural adverse events in patients with objectively confirmed OSA compared to controls [83-86]. And in a small prospective case-control study of patients with PSGconfirmed OSA $(n=31)$ undergoing colonoscopy, there were no differences in the rates of adverse events despite higher midazolam dosing and deeper sedation in the OSA patients [87]. Together, these findings suggest that the level of sedation achieved with conscious sedation, in the absence of general anesthesia and significant postprocedural opioid use, does not increase risk for peri-procedural cardiopulmonary complications in patients with OSA.

\section{Ambulatory Surgery}

There is a small but growing amount of data regarding intraand postoperative risks in OSA patients undergoing ambulatory surgery. Studies published to date suggest that unanticipated admission is not increased in patients with known or suspected OSA [88-92]. Transient hypoxemia has been described in some patients postoperatively in two observational cohort studies, though the lack of control groups, as well as the lack of changes in other outcomes, makes the relevance of these findings debatable $[90,91]$. In addition, one of the studies also found an increased likelihood of difficult intubation, intraoperative use of pressors, and lower postoperative PACU oxygen saturation in patients at high risk for OSA [89]; however, the clinical significance of these findings is not clear. The Society for Ambulatory Anesthesia (SAMBA) recently published recommendations for patients with suspected or known OSA stating that these patients could be considered for ambulatory surgery as long as co-morbidities were optimized, CPAP therapy was able to be used postoperatively (if known OSA), and postoperative opioids were minimized [93]. More recently, a review of ambulatory surgery in OSA patients echoed these recommendations but also called for further research to better phenotype OSA patients to identify those who may benefit from prolonged postoperative monitoring [51].

\section{Elective Inpatient Surgery}

Perioperative complications related to OSA can occur both in the operating suite as well as postoperatively.

\section{Intraoperative Complications}

Most [89, 94-97] but not all [98, 99] studies have found an association between OSA and difficult airway management. The strongest association appears to be in those with severe OSA (OR 4.46) [96]. While most of the studies examining this question are small to moderate in size, two very large database studies did confirm this relationship though they are limited methodically by how OSA was defined ("chart diagnosis") $[94,96]$. The overall quality of the studies investigating airway management in OSA patients is felt to be low [80••] and additional work is needed.

Conversely, unanticipated difficulty with intubation in a surgical patient may be predictive of undiagnosed OSA and further sleep evaluation should be considered [100]. In addition to airway management problems, more intraoperative pressor use may be required in patients with known or suspected OSA [89].

\section{Postoperative Complications}

A wide range of postoperative complications have been reported in OSA patients undergoing a variety of surgical interventions. Complications reported vary by study and are listed in Table 1. Significant heterogeneity exists between studies regarding methodology for diagnosing OSA, the types of populations studied, the surgical interventions performed, and the outcomes reported (as well as methods of measuring outcomes), which may help to explain some of the inconsistency in the findings $[80 \bullet \bullet$.

\section{Pulmonary Complications}

A spectrum of postoperative pulmonary complications have been described in patients with known or suspected OSA. Respiratory failure following surgery is perhaps the most concerning adverse event as this may lead to emergent reintubation or the urgent need for noninvasive ventilation, along with transfer to a higher acuity of care. While some studies have specifically reported on this outcome [22, 101, $102,103 \bullet, 104,105]$, many have combined pulmonary complications into composite measures making it difficult to sort out the associations. With two exceptions [22, 102], studies reporting on postoperative respiratory failure have found an association between a diagnosis of OSA and higher rates of 
Table 1 Postoperative complications reported in studies of OSA patients

\begin{tabular}{ll}
\hline Category & Postoperative complication \\
\hline Pulmonary & Hypoxia \\
& Acute hypercapnia \\
& Pneumonia \\
& Atelectasis \\
& Bronchospasm \\
& Adult respiratory distress syndrome \\
& Pulmonary embolism \\
& Need for urgent noninvasive ventilation \\
& Need for reintubation \\
& Arrhythmias \\
Cardiac & Ischemia \\
& Myocardial infarction \\
& Pulmonary edema \\
Cardiac arrest \\
Delirium \\
Encephalopathy \\
Stroke \\
Gastrointestinal bleeding \\
Infection \\
Mortality \\
Unplanned transfer to higher acuity of care \\
Length of stay \\
\end{tabular}

this complication (OR 1.6-4.3, all statistically significant). The studies reporting an association have used PSG confirmation $[104,105]$ or ICD coding $[101,103 \cdot]$ to define OSA, whereas one of the studies that did not find an association used a screening questionnaire to define low and high risk for OSA populations [22]. The other study not finding an association with respiratory failure and an ICD code for OSA noted much higher rates of reintubation (OR 4.4) and CPAP/noninvasive ventilation use (OR 14.1), but not respiratory failure, which suggests potential methodological issues affecting the analysis [102]. One study that deserves specific mention is the large database study utilizing the national inpatient sample ( $n>1$ million) that examined a variety of different surgical procedures [103•]. In this study, the association between postoperative respiratory failure and OSA was strongest in patients undergoing orthopedic or urologic surgery but weakest (and not significant) in patients subjected to abdominal surgery, indicating the type of surgery may play a role in outcomes as well. Furthermore, emergent reintubation occurred earlier in the postoperative course in patients who had OSA compared to those without a diagnosis of OSA. This begs the questions of whether or not enhanced monitoring and/or a lower threshold for reintubating patients with OSA influenced these findings.
Studies examining the outcome of "combined pulmonary complications" are difficult to interpret as hypoxemia and hypercapnia are often listed as adverse events in the composite outcome and the clinical relevance of these findings is questionable, as discussed below. Some studies have reported specifically on pneumonia and pulmonary embolism as outcomes [22, 101, 102, 105-109]. There is no clear consensus regarding the association of pneumonia and OSA in the perioperative setting as some studies report an association (general surgery, orthopedic surgery) $[101,105,109]$ whereas others do not (bariatric surgery, orthopedic surgery) [22, 102, 108]. Likewise, the association of postoperative pulmonary embolism and OSA remains uncertain as two studies noted higher rates of pulmonary embolism in OSA patients (both orthopedic surgery) $[101,106]$ and two studies did not (general surgery, orthopedic surgery - though this study was shoulder surgery) $[101,107]$. Further research is warranted to better clarify the association between OSA and these relevant outcomes.

Postoperative hypoxemia and desaturations have frequently been reported as outcomes in studies examining OSA in the perioperative setting [21, 23-25, 61, 104, 110]. Most studies show an association between OSA and more frequent postoperative hypoxemia and/or desaturation. While hypoxemia is felt to be one of the mechanisms leading to serious adverse consequences in the postoperative period, the clinical significance of this is a matter of debate as patients with OSA frequently experience hypoxemia and desaturations at baseline during sleep $[6,17,20]$. Most recent studies are no longer reporting this as an outcome of interest.

\section{Cardiovascular Complications}

Much like pulmonary complications, most studies reporting on adverse postoperative cardiovascular consequences have reported these events (cardiac arrest, ischemia, myocardial infarction, heart failure, arrhythmias) as a single combined outcome [21, 106, 107, 109-112]. With one exception [109], all studies have reported no association between OSA and more frequent composite cardiovascular complications. A variety of methodologies were used and a wide spectrum of surgical procedures were evaluated in these analyses. Two studies reporting specifically on postoperative myocardial infarction also failed to find an association with a diagnosis of OSA $[25,104]$.

Conversely, a growing number of studies have examined the association between OSA and atrial fibrillation in the postoperative setting $[27,102,103 \bullet, 113]$. These studies have reported higher rates of atrial fibrillation in patients with known or suspected OSA [27, 102, 103•, 113]. A recent meta-analysis suggests the correlation between OSA and 
postoperative atrial fibrillation may be most relevant for patients undergoing coronary artery bypass surgery [114].

Special mention is warranted regarding a large administrative database study where patients had a PSG-confirmed diagnosis of OSA before (OSA group) or after (undiagnosed OSA group) their elective surgery and were compared to a control group for cardiovascular outcomes [105]. In this study, the OSA group, who was also provided with a prescription for CPAP (though use could not be verified), had the same rates of cardiac arrest and shock following surgery as the control group, while the undiagnosed OSA group experienced statistically significantly higher rates than the control group (OR 2.40). This implies that treatment of OSA could potentially attenuate the rates of postoperative cardiac arrest and shock while unrecognized OSA may increase the frequency of this adverse outcome. While there are methodological issues with this study, it is provocative and additional work in this area is needed.

\section{Neurologic Complications}

Postoperative encephalopathy and delirium represent challenging problems for perioperative-care providers. While multiple factors have been associated with neurocognitive dysfunction following surgery, mounting evidence indicates that OSA may be an important factor. Two retrospective studies included encephalopathy [112] and delirium [115•] in their outcome measures, both finding an association with OSA. More recently, two prospective studies utilizing PSG- or HSAT-confirmed diagnoses of OSA and a standardized definition of postoperative delirium have found significant correlations between OSA and the incidence of delirium after surgery [116, 117]. One investigation found the rate of delirium after orthopedic surgery to be increased 2.5 fold if OSA was present [117], while the other noted a sixfold increase in delirium following cardiac surgery in patients with an $\mathrm{AHI} \geq 19$ [116]. Neither study noted a correlation with hypoxemia and incidence of delirium. Whether treatment of OSA prior to surgery could reduce postoperative delirium remains to be demonstrated.

Interestingly, postoperative stroke and its association with OSA has not been sufficiently examined to assess whether a relationship exists or not. An ongoing large prospective study examining OSA and postoperative vascular outcomes may shed some light on this topic [118].

\section{Miscellaneous Complications}

Postoperative infections have been associated with OSA in a retrospective single-center study of cardiac surgery patients [112] though this has not been reported by others. Similarly, OSA has been linked to postoperative bleeding in a small single-center study [19], but it would be difficult to explain mechanistically why this would occur. Contrary to the investigator's hypotheses, two studies evaluating wound healing in OSA patients found better wound healing in those diagnosed with or at risk for OSA [91, 119]. Ischemic preconditioning has been proposed as a possible mechanism for these latter findings $[80 \bullet \cdot$.

\section{Mortality}

With early case reports documenting deaths in OSA patients following surgery $[5,120]$, and the legal literature describing this as well [121], it has been assumed that, through the mechanisms discussed above, OSA would be associated with increased postoperative mortality. However, more recent research suggests this remains an unsettled issue. The latest systematic review of perioperative OSA studies found 13 studies reporting on mortality as an outcome [80••], and only one of these studies reported that OSA was associated with increased postoperative in-hospital mortality [106]. The majority of the remaining studies did not find an association, though three studies reported lower postoperative mortality following surgery on OSA patients $[102,103$, 122]. It has been hypothesized that obesity and ischemic preconditioning in OSA patients could have protective effects leading to reduced postoperative mortality [103•]. In addition, as many of the adverse respiratory outcomes occurred early in the patients' hospital stays, and may have been related to postoperative opioid use in OSA patients, it has been theorized that early intervention and treatment of this reversible problem may have led to improved outcomes in these patients [103•]. It is also plausible that, since all the studies describing lower postoperative mortality in OSA patients were large database studies using ICD-based diagnoses of OSA, the control groups may have had substantial numbers of undiagnosed and unrecognized OSA patients (as studies would support $[12,13]$ ) who may be particularly vulnerable to adverse events following surgery due to the lack of closer monitoring or other maneuvers hoped to reduce risk.

\section{Resource Utilization}

Criteria used to describe resource utilization have included unplanned admission, unanticipated ICU admission, ICU resource use, length of stay (LOS), and hospital charges. Likely due to variable methodologies for defining OSA, differing surgical populations, and heterogeneous practice patterns, a consistent finding regarding resource utilization by OSA patients following surgery has been difficult to establish. In the largest systematic review to date to examine this topic, 11 
studies found higher resource utilization in OSA patients versus eight studies that did not $[80 \bullet \cdot]$. As noted earlier, rates of unplanned admissions following ambulatory surgery have not been shown to be higher for patients with OSA [88-92]. Critical care resource requirements may be more significant in surgical patients with OSA, though few studies have measured this outcome [25, 109, 123-125]. Overall hospital LOS has not been consistently found to be higher in OSA patients following surgery as eight studies have noted longer LOS [23, $103 \cdot, 104,107,109,115 \bullet, 123,126]$ whereas six studies have not $[92,102,103 \cdot, 106,110,112]$. Furthermore, two of these studies reported a shorter postoperative LOS in patients with OSA $[103 \bullet, 106]$. While not fully understood, it has been proposed that this finding may be related to some of the factors mentioned above contributing to a possibly lower postoperative mortality rate [103•]. And finally, overall hospital charges have been described as being higher $[103 \bullet, 106]$ and lower $[102,103 \cdot, 107]$ in patients with OSA undergoing surgery.

\section{Conclusions}

Patients with OSA subjected to surgery are at increased risk for a wide variety of complications in the perioperative time period, with the strongest evidence supporting difficulty with intubations and higher rates of postoperative respiratory failure, atrial fibrillation, and delirium. While immediate perioperative complications are frequently the result of the negative effects of narcotics and sedatives or the residual effects of anesthesia, later events may be related to other factors such as REM-rebound sleep. Further research is warranted to investigate phenotypic differences between OSA patients and how this may impact risk for postoperative adverse events.

Anesthetic, sedative, and analgesic drugs should be used with caution in patients with OSA or in those suspected of having OSA. Other strategies to help reduce postoperative complications, such as enhanced monitoring and use of CPAP therapy, are currently under investigation. In the meantime, CPAP use and postoperative monitoring of OSA patients should be tailored to the specifics of each case, though a conservative approach seems prudent for patient safety.

\section{Compliance with Ethical Standards}

Conflict of Interest Dennis Auckley has received speaker honorarium from Cleveland Clinic Foundation outside of the submitted work.

Human and Animal Rights and Informed Consent This article does not contain any studies with human or animal subjects performed by any of the authors.

\section{References}

Papers of particular interest, published recently, have been highlighted as:

- Of importance

- Of major importance

1. Peppard PE, Young T, Barnet JH, Palta M, Hagen EW, Hla KM. Increased prevalence of sleep-disordered breathing in adults. Am J Epidemiol. 2013;177:1006-14. This updated epidemiologic study from the Wisconsin Sleep Cohort Study provides the most recent and accurate prevalence estimates for OSA in the general population.

2. Redline S, Sotres-Alvarez D, Loredo J, Hall M, Patel SR, Ramos A, et al. Sleep-disordered breathing in Hispanic/Latino individuals of diverse backgrounds. The Hispanic Community Health Study/ Study of Latinos. Am J Respir Crit Care Med. 2014;189(3):33544.

3. Senthilvel E, Auckley D, Dasarathy J. Evaluation of sleep disorders in the primary care setting: history taking compared to questionnaires. J Clin Sleep Med. 2011;7(1):41-8.

4. Stansbury RC, Stollo PJ. Clinical manifestations of sleep apnea. J Thorac Dis. 2015;7(9):E298-310.

5. Rennotte MT, Baele P, Aubert G, Rodenstein D. Nasal continuous positive airway pressure in the perioperative management of patients with obstructive sleep apnea submitted to surgery. Chest. 1995;107:367-74.

6. Loube DI, Erman MK, Reed W. Perioperative complications in obstructive sleep apnea patients. Sleep Breath. 1997;2(1):3-10.

7. American Academy of Sleep Medicine. International classification of sleep disorders. 3rd ed. Darien: American Academy of Sleep Medicine; 2014.

8. Epstein LJ, Kriso D, Strollo PJ, Friedman N, Malhotra A, Patil SP, et al. Clinical guideline for the evaluation, management and longterm care of obstructive sleep apnea in adults. J Clin Sleep Med. 2009;5(3):263-76.

9. https://www.cms.gov/medicare-coverage-database/details/ncadecision-memo. aspx ?NCAId $=227 \&$ ver $=11 \&$ NcaName $=$ Sleep + Testing+for+Obstructive + Sleep+Apnea+(OSA) $\&$ CoverageSelection $=$ National $\&$ KeyWord $=$ sleep + testing \&KeyWordLookUp=Title\&KeyWordSearchType $=$ And $\& b c=$ gAAAACAAEAAA\&. Accessed 15 Feb 16.

10. Berry RB, Brooks R, Gamaldo CE, Harding SM, Lloyd RM, Marcus CL, Vaughn BV; for the American Academy of Sleep Medicine. The AASM manual for the scoring of sleep and associated events: rules, terminology and technical specifications, version 2.2. www.aasmnet.org. Darien, Illinois: American Academy of Sleep Medicine; 2015.

11. Kuna ST, Gurubhagavatula I, Maislin G, Hin S, Hartwig KC, McCloskey S, et al. Noninferiority of functional outcome in ambulatory management of obstructive sleep apnea. Am J Respir Crit Care Med. 2011;183(9):1238-44.

12. Finkel KJ, Searleman AC, Tymkew H, Tanaka CY, Saager L, Safer-Zadeh E, et al. Prevalence of undiagnosed obstructive sleep apnea among adult surgical patient's in an academic medical center. Sleep Med. 2009;10(7):753-8.

13. Singh M, Liao P, Kobah S, Wijeysundera DN, Shapiro C, Chung F. Proportion of surgical patients with undiagnosed obstructive sleep apnea. Br J Aneaesth. 2013;110(4):629-36.

14. Liao P, Luo Q, Elsaid H, Kang W, Shapiro C, Chung F. Peroperative auto-titrated continuous positive airway pressure treatment in surgical patients with obstructive sleep apnea: a randomized controlled trial. Anesthesiology. 2013;119(4):837-47. 
15. Chung F, Liao P, Sun Y, Amirshahi B, Fazel H, Shapiro CM, et al. Perioperative practical experiences in using a level 2 portable polysomnography. Sleep Breath. 2011;15(3):367-75.

16. Chung F, Liao P, Elsaid H, Islam S, Shapiro CM, Sun Y. Oxygen desaturation index from nocturnal oximetry: a sensitive and specific tool to detect sleep disordered breathing in surgical patients. Anesth Anal. 2012;114:993-1000.

17. Hang LW, Wang HL, Chen JH, Hsu JC, Lin HH, Chung WS, et al. Validation of overnight oximetry to diagnose patients with moderate to severe obstructive sleep apnea. BMC Pulm Med. 2015;15: 24.

18. Ramsey R, Mehra R, Strohl KP. Variations in physician interpretation of overnight pulse oximetry monitoring. Chest. 2007;132: $852-9$.

19. Hwang D, Shakir N, Limann B, Sison C, Kalra S, Shulman L, et al. Association of sleep-disordered breathing with postoperative complications. Chest. 2008;133:1128-34.

20. Chung F, Zhou L, Liao P. Parameters from preoperative overnight oximetry predict postoperative adverse events. Minerva Anestesiol. 2014;80(10):1084-95.

21. Gali B, Whalen FX, Schroeder DR, Gay PC, Plevak DJ. Identification of patients at risk for postoperative respiratory complications using a preoperative obstructive sleep apnea screening tool and postanesthesia care assessment. Anesthesiology. 2009;110:869-77.

22. Ursavas A, Guven T, Coskun F, Ege E, Yilmazlar A. Association between self-reported snoring, STOP questionnaire and postoperative pulmonary complications in patients submitted to orthophaedic surgery. Multidiscip Respir Med. 2013;8:3.

23. Berend KR, Ajluni AF, Núñez-García LA, Lombardi AV, Adams JB, Nunez-Garcia LA, et al. Prevalence and management of obstructive sleep apnea in patients undergoing total joint arthroplasty. J Arthroplasty. 2010;25:54-7.

24. Eikermann M, Garzon-Serrano J, Kwo J, Grosse-Sundrup M, Schmidt U, Bigatello L. Do patients with obstructive sleep apnea have an increased risk of desaturation during induction of anesthesia for weight loss surgery? Open Respir Med J. 2010;4:58-62.

25. Munish M, Sharma V, Yarussi KM, Sifain A, Porhomayon J, Nader N. The use of practice guidelines by the American Society of Anesthesiologists for the identification of surgical patients at high risk of sleep apnea. Chron Respir Dis. 2012;9:22130 .

26. Mungan U, Ozeke O, Mavioglu L, Ertan C, Karaca IO, Keskin $\mathrm{G}$, et al. The role of the preoperative screening of sleep apnoea by Berlin Questionnaire and Epworth Sleepiness Scale for postoperative atrial fibrillation. Heart Lung Circ. 2013;22:38-42.

27. van Oosten EM, Hamilton A, Petsikas D, Payne D, Redfearn DP, Zhang S, et al. Effect of preoperative obstructive sleep apnea on the frequency of atrial fibrillation after coronary artery bypass grafting. Am J Cardiol. 2014;113:919-23.

28. Vasu TS, Doghramji K, Cavallazzi R, Grewal R, Hirani A, Leiby $\mathrm{B}$, et al. Obstructive sleep apnea syndrome and postoperative complications: clinical use of the STOP-BANG questionnaire. Arch Otolaryngol Head Neck Surg. 2010;136:1020-4.

29. Lockhart EM, Willingham MD, Ben AA, Helsten DL, Bedair BA, Thomas J, et al. Obstructive sleep apnea screening and postoperative mortality in a large surgical cohort. Sleep Med. 2013;14: 407-15.

30. Ramachandran SK, Josephs LA. A meta-analysis of clinical screening tests for obstructive sleep apnea. Anesthesiology. 2009;110:928-39.

31. Abrishami A, Khajehdehi A, Chung F. A systematic review of screening questionnaires for obstructive sleep apnea. Can J Anaesth. 2010;57:423-38.
32. Chung F, Subramanyam R, Liao P, Sasaki E, Shapiro C, Sun Y. High STOP-Bang score indicates a high probability of obstructive sleep apnoea. Br J Anaesth. 2012;108:768-75.

33. Farney RJ, Walker BS, Farney RM, Snow GL, Walker JM. The STOP-Bang equivalent model and prediction of severity of obstructive sleep apnea: relation to polysomnographic measurements of the apnea/hypopnea index. J Clin Sleep Med. 2011;7:459-67.

34. Nagappa M, Liao P, Wong J, Auckley D, Ramachandran SK, Memtsoudis S, et al. Validation of the STOP-Bang questionnaire as a screening tool for obstructive sleep apnea among different populations: a systematic review and meta-analysis. PLoS One. 2015;10(12):e0143697.

35. McIsaac DL, Gershon A, Wijeysundera D, Bryson GL, Badner N, van Walraven C. Identifying obstructive sleep apnea in administrative data: a study of diagnostic accuracy. Anesthesiology. 2015;123(2):253-63.

36. Jing J, Huang T, Cui W, Shen H. Effect on quality of life of continuous positive airway pressure in patients with obstructive sleep apnea syndrome: a meta-analysis. Lung. 2008;186(3):13144.

37. McNicholas WT. Cardiovascular outcomes of CPAP therapy in obstructive sleep apnea syndrome. Am J Physiol Regul Integr Comp Physiol. 2007;293(4):R1666-70.

38. Mehta V, Subramanyam R, Shapiro CM, Chung F. Health effects of identifying patients with undiagnosed obstructive sleep apnea in preoperative clinic: a follow-up study. Can J Anaesth. 2012;59(6):544-55

39. Lee W, Nagubadi S, Kryger MH, Mokhlesi B. Epidemiology of obstructive sleep apnea: a population-based perspective. Expert Rev Respir Med. 2008;2(3):349-64.

40. Chung F, Yegneswaran B, Liao P, et al. Validation of the Berlin Questionnaire and American Society for Anesthesiologists checklist as screening tools for obstructive sleep apnea in surgical patients. Anesthesiology. 2008;108:822-30.

41. Roggenbach J, Saur P, Hofer S, Bruckner T, Preusch M, Carbone $\mathrm{R}$, et al. Incidence of perioperative sleep-disordered breathing in patients undergoing major surgery: a prospective cohort study. Patient Saf Surg. 2014;8:13-8.

42. Foldvary-Schaefer N, Kaw R, Collop N, Andrews ND, Bena J, Wang L, et al. Prevalence of undetected sleep apnea in patients undergoing cardiovascular surgery and impact on postoperative outcomes. J Clin Sleep Med. 2015;11(10):1083-9.

43. Frey WC, Pilcher J. Obstructive sleep-related breathing disorders in patients evaluated for bariatric surgery. Obes Surg. 2003;13(5): 676-83.

44. Hallowell PT, Stellato TA, Schuster M, Graf K, Robinson A, Crouse $\mathrm{C}$, et al. Potentially life-threatening sleep apnea is unrecognized without aggressive evaluation. Am J Surg. 2007;193(3): 364-7.

45. Sareli AE, Cantor CR, Williams NN, Korus G, Raper SE, Pien G, et al. Obstructive sleep apnea in patients undergoing bariatric surgery - a tertiary center experience. Obes Surg. 2011;21(3):31627.

46. Peromaa-Haavisto P, Tuomilehto H, Kössi J, Virtanen J, Luostarinen M, Pihlajamäki J, et al. Prevalence of obstructive sleep apnoea among patients admitted for bariatric surgery. A prospective multicentre trial. Obes Surg. 2015.

47. Hillman DR, Platt PR, Eastwood PR. Anesthesia, sleep and upper airway collapsibility. Anesthesiol Clin. 2010;28:44355.

48. Ankichetty S, Wong J, Chung F. A systematic review of the effects of sedatives and anesthetics in patients with obstructive sleep apnea. J Anaesthesiol Clin Pharmacol. 2011;27(4):447-58. Welldone systematic review on the impact of sedatives and anesthetics on OSA patients in the perioperative setting. 
49. Berry RB, Kouchi K, Bower JL, Light RW. Effect of upper airway anesthesia on obstructive sleep apnea. Am J Respir Crit Care Med. 1995;151:1857-61.

50. Berry RB, McNellis MI, Kouchi K, Light RW. Upper airway anesthesia reduces phasic genioglossus activity during sleep apnea. Am J Respir Crit Care Med. 1997;156(1):127-32.

51. Stierer TL. Collop NA perioperative assessment and management for sleep apnea in the ambulatory surgical patient. Chest. 2015;148(2):559-65.

52. Richard W, Kox D, den Herder C, Laman M, van Tinteren $\mathrm{H}$, de Vries $\mathrm{N}$. The role of sleep position in obstructive sleep apnea syndrome. Eur Arch Otorhinolaryngol. 2006;263(10):946-50.

53. Stadler DL, McEvoy RD, Bradley J, Paul D, Catcheside PG. Changes in lung volume and diaphragm muscle activity at sleep onset in obese obstructive sleep apnea patients vs. healthy-weight controls. J Appl Physiol. 2010;109(4):1027-36.

54. Pelosi P, Croci M, Calappi E, Mulazzi D, Cerisara M, Vercesi P, et al. Prone positioning improves pulmonary function in obese patients during general anesthesia. Anesth Analg. 1996;83(3):57883.

55. Karcz M, Papadakos PJ. Respiratory complications in the postanesthesia care unit: a review of pathophysiological mechanisms. Can J Respir Ther. 2013;49(4):21-9.

56.• Lam KK, Kunder S, Wong J, Doufas A, Chung F. Obstructive sleep apnea, pain and opioids: is the riddle solved? Curr Opin Anesthesiol. 2016;29:134-40. This is an excellent review of the effects of opioids on ventilation, OSA, pain thresholds, and sleep itself. It highlights the current state of knowledge and raises some intriguing points.

57. Ramachandran SK, Haider N, Saran KA, Mathis M, Kim J, Morris $\mathrm{M}$, et al. Life-threatening critical respiratory events: a retrospective study of postoperative patients found unresponsive during analgesic therapy. J Clin Anesth. 2011;23(3):207-13.

58. Lee LA, Caplan RA, Stephens LS, Posner KL, Terman GW, Voepel-Lewis T, et al. Postoperative opioid-induced respiratory depression: a closed claims analysis. Anesthesiology. 2015;122(3):659-65.

59. Koo CY, Eikermann M. Respiratory effects of opioids in perioperative medicine. Open Anesthesiol. 2011;5(Suppl 1-M6):23-4.

60. Stuth EA, Stucke AG, Zuperku EJ. Effects of anesthetics, sedatives, and opioids on ventilatory control. Compr Physiol. 2012;2(4):2281-367.

61. Chung F, Liao P, Yegneswaran B, Shapiro CM, Kang W. Postoperative changes in sleep-disordered breathing and sleep architecture in patients with obstructive sleep apnea. Anesthesiology. 2014;120(2):287-98.

62. Doufas AG, Tian L, Padrez KA, Suwanprathes P, Cardell JA, Maecker HT, et al. Experimental pain and opioid analgesia in volunteers at high risk for obstructive sleep apnea. PLoS One. 2013;8(1):e54807.

63. Khalid I, Roehrs TA, Hudgel DW, Roth T. Continuous positive airway pressure in severe obstructive sleep apnea reduces pain sensitivity. Sleep. 2011;34(12):1687-91.

64. Mokhlesi B, Punjabi NM. "REM-related" obstructive sleep apnea: an epiphenomenon or a clinically important entity? Sleep. 2012;35(1):5-7.

65. Knill RL, Moote CA, Skinner MI, Rose EA. Anesthesia with abdominal surgery leads to intense REM sleep during the first postoperative week. Anesthesiology. 1990;73:52-61.

66. Rosenberg J, Wildschiødtz G, Pedersen MH, von Jessen F, Kehlet H. Late postoperative nocturnal episodic hypoxaemia and associated sleep pattern. Br J Anaesth. 1994;72(2):145-50.

67. Liak C, Fitzpatrick M. Coagulability in obstructive sleep apnea. Can Respir J. 2011;18(6):338-48.
68. Somers VK, Dyken ME, Mark AL, Abboud FM. Parasympathetic hyperresponsiveness and bradyarrhythmias during apnoea in hypertension. Clin Auton Res. 1992;2(3):171-6.

69. Mehra R, Benjamin EJ, Shahar E, Gottlieb DJ, Nawabit R, Kirchner HL, et al. Association of nocturnal arrhythmias with sleep-disordered breathing: The Sleep Heart Health Study. Am J Respir Crit Care Med. 2006;173(8):910-6.

70. Gami AS, Howard DE, Olson EJ, Somers VK. Day-night pattern of sudden death in obstructive sleep apnea. N Engl J Med. 2005;352:1206-14.

71. Bucks RS, Olaithe M, Eastwood P. Neurocognitive function in obstructive sleep apnoea: a meta-review. Respirology. 2013;18(1):61-70.

72. Mirrakhimov AE, Brewbaker CL, Krystal AD, Kwatra MM. Obstructive sleep apnea and delirium: exploring possible mechanisms. Sleep Breath. 2014;18(1):19-29.

73. Steiner L. Postoperative delirium. Part 2: detection, prevention and treatment. Eur J Anaesthesiol. 2011;28(10):723-32.

74. Bateman BT, Eikermann M. Obstructive sleep apnea predicts adverse perioperative outcome: evidence for an association between obstructive sleep apnea and delirium. Anesthesthiology. 2012;116:753-5.

75. Eckert DJ, Younes MK. Arousal from sleep: implications for obstructive sleep apnea pathogenesis and treatment. J Appl Physiol. 2014;116(3):302-13.

76. Sands SA, Eckert DJ, Jordan AS, Edwards BA, Owens RL, Butler JP, et al. Enhanced upper-airway muscle responsiveness is a distinct feature of overweight/obese individuals without sleep apnea. Am J Respir Crit Care Med. 2014;190(8):930-7.

77. Kaw R, Chung F, Pasupuleti V, Mehta J, Gay P, Hernandez AV. Meta-analysis of the association between obstructive sleep apnea and postoperative outcome. Br J Anaesth. 2012;109(6):897-906.

78. Vasu TS, Grewal R, Doghramji K. Obstructive sleep apnea syndrome and perioperative complications: a systematic review of the literature. J Clin Sleep Med. 2012;8(2):199-207.

79. Hai F, Porhomayon J, Vermont L, Frydrych L, Jaoude P, El-Solh AA. Postoperative complications in patients with obstructive sleep apnea: a meta-analysis. J Clin Anesth. 2014;26(8):591-600.

80.• Opperer M, Cozowicz C, Bugada D, Mokhlesi B, Kaw R, Auckley D, et al. Does obstructive sleep apnea influence perioperative outcomes? A qualitative systematic review. Anesth Analg. 2016; in press. Most recent and comprehensive review looking at the association between OSA and postoperative outcomes.

81. Cote GA, Hovis CE, Hovis RM, Waldbaum L, Early DS, Edmundowicz SA, et al. A screening instrument for sleep apnea predicts airway maneuvers in patients undergoing advanced endoscopic procedures. Clin Gastroenterol Hepatol. 2010;8(8):660-5.

82. Mador MJ, Nadler J, Mreyoud A, Khadka G, Gottumukkala VA, Abo-Khamis M, et al. Do patients at risk of sleep apnea have an increased risk of cardio-respiratory complications during endoscopy procedures? Sleep Breath. 2012;16(3):609-15.

83. Adler DG, Kawa C, Hilden K, Fang J. Nurse-administered propofol sedation is safe for patients with obstructive sleep apnea undergoing routine endoscopy. Dig Dis Sci. 2011;56(9):2666-71.

84. Gill J, Vidyarthi G, Kulkarni P, Anderson W, Boyd W. Safety of conscious sedation in patients with sleep apnea in a veteran population. South Med J. 2011;104(3):185-8.

85. Mador MJ, Abo-Khami M, Nag N, Mreyoud A, Jallu S, Mehboob $\mathrm{S}$. Does sleep apnea increase the risk of cardiorespiratory complications during endoscopy procedures? Sleep Breath. 2011;15: 393-401.

86. Mudambi L, Speigelman A, Geron D, Hirshkowitz M, Mokhlesi B, Shaib Y, et al. Obstructive sleep apnea is not associated with higher health care utilization after colonoscopy under conscious sedation. Ann Am Thorac Soc. 2016; in press. 
87. Cha JM, Jeun JW, Pack KM, Lee JI, Joo KR, Shin HP, et al. Risk of sedation for diagnostic esophagogastroduodenoscopy in obstructive sleep apnea patients. World J Gastroenterol. 2013;19(29):4745-51.

88. Sabers C, Plevak DJ, Schroeder DR, Warner DO. The diagnosis of obstructive sleep apnea as a risk factor for unanticipated admissions in outpatient surgery. Anesth Analg. 2003;96(5):1328-35.

89. Stierer TL, Wright C, George A, Thompson RE, Wu CL, Collop $\mathrm{N}$. Risk assessment of obstructive sleep apnea in a population of patients undergoing ambulatory surgery. J Clin Sleep Med. 2010;6(5):467-72.

90. Liu SS, Chisholm MF, John RS, Ngeow J, Ma Y, Memtsoudis SG. Risk of postoperative hypoxemia in ambulatory orthopedic surgery patients with diagnosis of obstructive sleep apnea: a retrospective observational study. Patient Saf Surg. 2010;4(1):9-15.

91. Kurrek MM, Cobourn C, Wojtasik Z, Kiss A, Dain SL. Morbidity in patients with or at high risk for obstructive sleep apnea after ambulatory laparoscopic gastric banding. Obes Surg. 2011;21(10):1494-8.

92. Bryson GL, Gomez CP, Jee RM, Blackburn J, Taljaard M, Forster AJ. Unplanned admission after day surgery: a historical cohort study in patients with obstructive sleep apnea. Can J Anaesth. 2012;59(9):842-51.

93. Joshi GP, Ankichetty SP, Gan TJ, Chung F. Society for Ambulatory Anesthesia consensus statement on preoperative selection of adult patients with obstructive sleep apnea scheduled for ambulatory surgery. Anesth Analg. 2012;115(5):1060-8.

94. Kheterpal S, Martin L, Shanks AM, Tremper KK. Prediction and outcomes of impossible mask ventilation: a review of 50,000 anesthetics. Anesthesiology. 2009;110:891-7.

95. Iyer US, Koh KF, Chia NC, Macachor J, Cheng A. Perioperative risk factors in obese patients for bariatric surgery: a Singapore experience. Singap Med J. 2011;52:94-9.

96. Corso RM, Piraccini E, Calli M, Berger M, Gorini MC, Agnoletti $\mathrm{V}$, et al. Obstructive sleep apnea is a risk factor for difficult endotracheal intubation. Minerva Anestesiol. 2011;77(1):99-100.

97. Kheterpal S, Healy D, Aziz MF, Shanks AM, Freundlich RE, Linton F, et al. Incidence, predictors, and outcome of difficult mask ventilation combined with difficult laryngoscopy: a report from the multicenter perioperative outcomes group. Anesthesiology. 2013;119:1360-9.

98. Vest D, Lee D, Newcome K, Stamper H. A retrospective review of difficult intubations: is obstructive sleep apnea a predictor? Clin Nurse Spec. 2013;27:128-31.

99. Neligan PJ, Porter S, Max B, Malhotra G, Greenblatt EP, Ochroch EA. Obstructive sleep apnea is not a risk factor for difficult intubation in morbidly obese patients. Anesth Analg. 2009;109(4): 1182-6.

100. Chung F, Yegneswaran B, Herrera F, et al. Patients with difficult intubation may need referral to sleep clinics. Anesth Analg. 2008;107:915-20.

101. Memtsoudis S, Liu SS, Ma Y, Chiu YL, Walz JM, Gaber-Baylis LK, et al. Perioperative pulmonary outcomes in patients with sleep apnea after noncardiac surgery. Anesth Analg. 2011;112:113-21.

102. Mokhlesi B, Hovda MD, Vekhter B, Arora VM, Chung F, Meltzer DO. Sleep-disordered breathing and postoperative outcomes after bariatric surgery: analysis of the nationwide inpatient sample. Obes Surg. 2013;23:1842-51.

103. Mokhlesi B, Hovda MD, Vekhter B, Arora VM, Chung F, Meltzer DO. Sleep-disordered breathing and postoperative outcomes after elective surgery: analysis of the nationwide inpatient sample. Chest. 2013;144:903-14. Large database study that examined multiple outcomes as related to ICD diagnosis of OSA, reported on reduced mortality in OSA patients, raising a number of questions.
104. Kaw R, Pasupuleti V, Walker E, Ramaswamy A, FoldvarySchafer N. Postoperative complications in patients with obstructive sleep apnea. Chest. 2012;141:436-41.

105. Mutter TC, Chateau D, Moffatt M, Ramsey C, Roos LL, Kryger M. A matched cohort study of postoperative outcomes in obstructive sleep apnea: could preoperative diagnosis and treatment prevent complications? Anesthesiology. 2014;121:707-18.

106. D'Apuzzo MR, Browne JA. Obstructive sleep apnea as a risk factor for postoperative complications after revision joint arthroplasty. J Arthroplasty. 2012;27:95-8.

107. Griffin JW, Novicoff WM, Browne JA, Brockmeier SF. Obstructive sleep apnea as a risk factor after shoulder arthroplasty. J Shoulder Elb Surg. 2013;22:e6-9.

108. Jensen C, Tejirian T, Lewis C, Yadegar J, Dutson E, Mehran A. Postoperative CPAP and BiPAP use can be safely omitted after laparoscopic Roux-en-Y gastric bypass. Surg Obes Relat Dis. 2008;4:512-4.

109. Memtsoudis SG, Stundner O, Rasul R, Chiu Y-LL, Sun X, Ramachandran S-KK, et al. The impact of sleep apnea on postoperative utilization of resources and adverse outcomes. Anesth Analg. 2014;118:407-18.

110. Liao P, Yegneswaran B, Vairavanathan S, Zilberman P, Chung F. Postoperative complications in patients with obstructive sleep apnea: a retrospective matched cohort study. Can J Anaesth. 2009;56:819-28.

111. Mador MJ, Goplani S, Gottumukkala VA, El-Solh AA, Akashdeep K, Khadka G, et al. Postoperative complications in obstructive sleep apnea. Sleep Breath. 2013;17:727-34.

112. Kaw R, Golish J, Ghamande S, Burgess R, Foldvary N, Walker E. Incremental risk of obstructive sleep apnea on cardiac surgical outcomes. J Cardiovasc Surg. 2006;47:683-9.

113. Mooe T, Gullsby S, Rabben T, Eriksson P. Sleep-disordered breathing: a novel predictor of atrial fibrillation after coronary artery bypass surgery. Coron Artery Dis. 1996;7:475-8.

114. Qaddoura A, Kabali C, Drew D, van Oosten EM, Michael KA, Redfearn DP, et al. Obstructive sleep apnea as a predictor of atrial fibrillation after coronary artery bypass grafting: a systematic review and meta-analysis. Can J Cardiol. 2014;30(12):1516-22.

115. Gupta R, Parvizi J, Hanssen AD, Gay PC. Postoperative complications in patients with obstructive sleep apnea syndrome undergoing hip or knee replacement: a case-control study. Mayo Clin Proc. 2001;76:897-905. Landmark study bringing attention to the issue of postoperative complications in OSA patients through a well-done case-controlled study.

116. Roggenbach J, Klamann M, von Haken R, Bruckner T, Karck M, Hofer S. Sleep disordered breathing is a risk factor for delirium after cardiac surgery: a prospective cohort study. Crit Care. 2014;18:477-86.

117. Flink BJ, Rivelli SK, Cox EA, White WD, Falcone G, Vall TP, et al. Obstructive sleep apnea and incidence of postoperative delirium after elective knee replacement in the nondemented elderly. Anesthesiology. 2012;116(4):788-96.

118. Chan MT, Wang CY, Seet E, Tam S, Lai HY, Walker S, et al. Postoperative vascular complications in unrecognized Obstructive Sleep apnoea (POSA) study protocol: an observational cohort study in moderate-to-high risk patients undergoing noncardiac surgery. BMJ Open. 2014;4(1):e004097.

119. Andrews KL, Dib M, Shives TC, Hoskin TL, Liedl DA, Boon AJ. The effect of obstructive sleep apnea on amputation site healing. J Vasc Nurs. 2012;30:61-3.

120. Ostermeier A, Rozen M, Hautkappe M, Klock PA, Klafta JM. Three sudden postoperative respiratory arrests associated with epidural opioids in patients with sleep apnea. Anesth Analg. 1997;85: 452-60.

121. Fouladpour N, Jesudoss R, Bolden N, Shaman Z, Auckley D. Perioperative complications in obstructive sleep apnea patients 
undergoing surgery: a review of the legal literature. Anesth Analg. 2016;122(1):145-51.

122. Nguyen NT, Masoomi H, Laugenour K, Sanaiha Y, Reavis KM, Mills SD, et al. Predictive factors of mortality in bariatric surgery: data from the nationwide inpatient sample. Surgery. 2011;150: 347-51.

123. Stundner O, Chiu Y-LL, Sun X, Ramachandran S-KK, Gerner P, Vougioukas V, et al. Sleep apnoea adversely affects the outcome in patients who undergo posterior lumbar fusion: a population-based study. Bone Joint J. 2014;96-B(2):242-8.
124. Kamath AF, McAuliffe CL, Baldwin KD, Lucas JB, Kosseim LM, et al. Unplanned admission to the intensive care unit after total hip arthroplasty. J Arthroplasty. 2012;27:1022-7.

125. Chia P, Seet E, Macachor JD, Iyer US, Wu D. The association of pre-operative STOP-BANG scores with postoperative critical care admission. Anaesthesia. 2013;68:950-2.

126. Pereira H, Xará D, Mendonça J, Santos A, Abelha FJ, Xara D, et al. Patients with a high risk for obstructive sleep apnea syndrome: postoperative respiratory complications. Rev Port Pneumol. 2013;19:144-51. 